\title{
Analysis of the Impact of Urban Microclimate on Air Conditioning Load Control
}

\author{
Xiaoqing $\mathrm{Hu}^{1, \mathrm{a}}$, Beibei Wang ${ }^{1}$, Wenlu $\mathrm{Ji}^{2}$, Peifeng Shen ${ }^{2}$ and Peng Chen ${ }^{3}$ \\ ${ }^{1}$ Department of Electrical Engineering, Southeast University, No. 2 Sipailou, Nanjing 210096, Jiangsu, China \\ ${ }^{2}$ Nanjing power supply company, No. 1 Aoti street, Nanjing 210019, Jiangsu, China \\ ${ }^{3}$ Rugao power supply company, No. 18 Zhongshan Road, Rugao 226500, Jiangsu, China
}

\begin{abstract}
Due to the presence of urban heat island effect (UHIE), high humidity and other urban microclimate, temperature of city central area rises. This causes that the actual air-conditioning energy consumption (ACEC) in the urban central area is much higher than that in the suburbs. Load control of air-conditioners (ACs) is considered to be equivalent to a power plant of the same capacity, and it can greatly reduce the system pressure to peak load shift. In this paper, a simplified second order transfer function control model of ACs is presented, and its parameters will be influenced by the ambient temperature and urban microclimate. The temperature is obtained by using the temperature inversion algorithm of the heat island effect. Then, the heat index is calculated by combining temperature and humidity. The ambient temperature index of urban central area is modified based on the above microclimate, and the second order linear time invariant model of aggregated ACs is upgraded to the linear time varying model. Furthermore, the consequent parameter changes of the second order transfer function model are studied and the influence of urban microclimate on AC load control is analyzed. The proposed method is verified on numerical examples.
\end{abstract}

Keywords. Peak load shifting, urban microclimate, air conditioning, closed-loop control.

\section{Introduction}

Energy consumption is closely related to climate change [1]. There are obvious differences in the meteorological conditions of different regions in a city. The concept of microclimate is needed to characterize these independent meteorological conditions. Urban microclimate, represented by urban heat island effect, temperature and humidity effect, and cumulative effect, involves the climate characteristics between the near-ground atmosphere and the topsoil in a relatively small space. In the initial power load forecasting, when measuring the impact of the weather, only temperature was considered. Later it was found that the relative humidity, wind speed and cloud distribution will have a significant impact on the power load, especially relative humidity. Microclimate makes temperature of city central area rise, and causes that the actual air-conditioning energy consumption (ACEC) in the urban central area is much higher than that in the suburb.

During the hot summer days in a lot of cities, the energy consumed by air conditioner is $30-50 \%$ of the total electric power consumed, and this proportion even exceeds $50 \%$ in some central business districts [2]. Direct Load Control (DLC), which usually reduces the loads by controlling air conditioners of the residential users or the small-scale business users, is an important kind of incentive-based demand response. These kinds of loads have heat energy storage ability and can switch or change the control parameters in a short time. Such behavior will not cause obvious side effects on users. Meanwhile, they have the potential to balance system power [3]. Flexible loads can be incorporated into the normal operation of power system's dispatch by the demand response.

The impact of UHIE on ACEC has been widely studied. Ojima found that over the past decade, cooling load of the buildings in Tokyo has increased by $10 \%$ $-20 \%$ on average due to the heat island effect [4]. According to this growth rate, it is expected to increase by more than $50 \%$ in 2030 . M. stantamouris etc., pointed out that when the average heat island intensity of the Athens area is over $10^{\circ} \mathrm{C}$, the cooling load of buildings will stiffen to double its original one in summer, and the peak load will treble [5][6]. By analyzing the simulation data of the outdoor air temperature of residential area, $\mathrm{Li}$ Xianting believes that the summer cooling load of urban residential quarters is increased by $10 \%-35 \%$, affected by the heat island effect [7].

In most cases, the control scheme of TCLs is based on the idea of optimization. A scheduling decision model has an objective function to maximize the load aggregate interest and to minimize the actual output deviation of 
ACs. It includes a constraint condition that the indoor temperature has to be within the range of $\left[T_{\min }, T_{\max }\right]$ in order to ensure the user's comfort level [8][9]. In [10], the authors explored a simplified second order linear time invariant transfer function model by analyzing the duty cycle of the air conditioning group. They also proposed a closed-loop control strategy for air conditioners to be involved in direct load control. In order to achieve the desired control effect, the authors assumed that the outdoor temperature remains constant. This assumption makes the parameters of the transfer function control model remain unchanged. Based on this control model, further exploration is carried out in this paper.

In this paper, a simplified second order transfer function control model of ACs is presented, and an internal model control (IMC) algorithm is also used for aggregated air conditioning load control. The heat island intensity is obtained by using the temperature inversion algorithm of the heat island effect. Then, the heat index is calculated by combining temperature and humidity. Based on the above urban microclimate, the ambient temperature index of urban central area is modified, and the second order linear time invariant model of aggregated ACs is upgraded to the linear time varying model. The consequent parameter changes of the second order transfer function model are studied and the influence of urban microclimate on AC load control is analyzed.

\section{Ambient temperature index modify based on urban microclimate}

\subsection{Temperature inversion algorithm of heat island effect}

There are many limitations in the traditional methods of measuring the urban heat island effect, since they can not effectively study the characteristics of urban heat island from the perspective of space layout and internal structure. The development of modern remote sensing technology and geographic information systems provide the technical foundation to address these issues. Remote sensing information retrieval method is to carry out the surface temperature inversion by using the thermal infrared sensor, for the changes of the different wave band radiation values of urban targets. According to the radiative transfer equation, radiance of the entrance pupil of the satellite at a given wavelength location can be expressed as:

$$
L_{\lambda}^{a t \text {-sensor }}=\left[\varepsilon_{\lambda} B\left(\lambda, T_{s}\right)+\left(1-\varepsilon_{\lambda}\right) L_{\lambda}^{a t m \downarrow}\right] \tau_{\lambda}+L_{\lambda}^{a t m \uparrow}
$$

where $\varepsilon_{\lambda}$ is the surface emissivity, $T$ is the inverse of the land surface temperature (LST) $, L_{\lambda}^{a t m \downarrow}, L_{\lambda}^{a t m \uparrow}$ are respectively atmospheric radiation downstream and upstream radiation, $\tau_{\lambda}$ is total atmospheric transmittance. $B\left(\lambda, T_{s}\right)$, measured in $\mathrm{W} \mu \mathrm{m}^{-1} \mathrm{~m}^{-2} \mathrm{sr}^{-1}$, represents the emitted blackbody radiation when the temperature is $T_{s} . \quad B\left(\lambda, T_{s}\right)$ is calculated as:

$$
B\left(\lambda, T_{s}\right)=\frac{c_{1} \lambda^{-5}}{\exp \left(\frac{c_{2}}{\lambda T_{s}}\right)-1}
$$

where

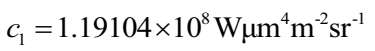
$c_{2}=1.43877 \times 10^{8} \mu \mathrm{mK}$. Based on the surface temperature inversion algorithm proposed by Jiménez-Muñoz and Sobrino11, the surface temperature can be approximated as:

$$
T_{s}=\gamma\left(\lambda, T_{0}\right)\left\{\varepsilon_{\lambda}^{-1}\left[\phi_{1}(\lambda, \omega) L_{\lambda}^{a t-\text { sensor }}+\phi_{2}(\lambda, \omega)\right]+\phi_{3}(\lambda, \omega)\right\}+\delta\left(\lambda, T_{0}\right)
$$

where $\gamma$ and $\delta$ are obtained by linear approximation of Plank equation. $\phi_{1}, \phi_{2}$ and $\phi_{3}$ is the state equation of the atmosphere calculated from the atmospheric state parameters, and can be expressed as follows:

$$
\begin{gathered}
\gamma\left(\lambda, T_{0}\right)=\frac{1}{\beta\left(\lambda, T_{0}\right)}, \quad \delta\left(\lambda, T_{0}\right)=-\frac{\alpha\left(\lambda, T_{0}\right)}{\beta\left(\lambda, T_{0}\right)} \\
\phi_{1}(\lambda, \omega)=\frac{1}{\tau(\lambda, \omega)} \\
\phi_{2}(\lambda, \omega)=-L^{a t n \downarrow}(\lambda, \omega)-\frac{L^{a t n \uparrow}(\lambda, \omega)}{\tau(\lambda, \omega)} \\
\phi_{3}(\lambda, \omega)=L^{a t n \downarrow}(\lambda, \omega), \lambda_{e f f}=\frac{\int_{\lambda_{1}}^{\lambda_{2}} \lambda f(\lambda) d \lambda}{\int_{\lambda_{1}}^{\lambda_{2}} f(\lambda) d \lambda}
\end{gathered}
$$

Where $\lambda_{\text {eff }}$ represents the effective wavelength of the sensor thermal infrared channel in $\mu \mathrm{m} . f(\lambda)$ is the spectral response function of the thermal infrared channel of the sensor. $\lambda_{1}, \lambda_{2}$ respectively indicate the start wavelength and the end wavelength, $\omega$ represents the atmospheric water vapor content in $\mathrm{g} / \mathrm{cm}^{2}$. In the above formula,

$$
\begin{gathered}
\alpha\left(\lambda, T_{0}\right)=B\left(\lambda, T_{0}\right)\left\{1-\frac{c_{2}}{T_{0}}\left[\frac{\lambda^{4}}{c_{1}} B\left(\lambda, T_{0}\right)+\frac{1}{\lambda}\right]\right\} \\
\beta\left(\lambda, T_{0}\right)=\frac{c_{2} B\left(\lambda, T_{0}\right)}{T_{0}^{2}}\left[\frac{\lambda^{4}}{c_{1}} B\left(\lambda, T_{0}\right)+\frac{1}{\lambda}\right]
\end{gathered}
$$

\subsection{The heat index calculated by combining temperature and humidity}

Unlike air temperature which tells how warm or cool the actual air around us is, apparent temperature tells us how warm or cool our bodies perceive the air to be. When the human body overheats, it cools itself by perspiring, or sweating; heat is then removed from the body by evaporation of that sweat. Humidity, however, slows the rate of this evaporation. The more moisture the surrounding air contains, the less moisture it's able to absorb from the skin's surface through evaporation. With less evaporation occurring, less heat is removed from the body, which is the reason why the air feels hotter than it really is when it's humid outside.

The heat index is a measure of how hot it feels outdoors as a result of air temperature and relative humidity combined. The computation of the heat index is a refinement of a result obtained by multiple regression analysis carried out by Lans P. Rothfusz12.

Heat Index $=-42.379+2.04901523 T+10.14333127 R H-0.22475541 T R H$

$$
\begin{aligned}
& -6.83783 \times 10^{-3} T^{2}-5.481717 \times 10^{-2} R H^{2}+1.22874 \times 10^{-3} T^{2} R H \\
& +8.5282 \times 10^{-4} T R H^{2}-1.99 \times 10^{-6} T^{2} R H^{2}
\end{aligned}
$$

where $T$ is temperature in degrees $\mathrm{F}$ and $R H$ is relative humidity in percent. $H I$ is the heat index expressed as an apparent temperature in degrees $\mathrm{F}$. If the $R H$ is less than 
$13 \%$ and the temperature is between 80 and 112 degrees $\mathrm{F}$, then the following adjustment is subtracted from $H I$ :

$$
\text { ADJUSTMENT }=[(13-R H) / 4] * \sqrt{(17-|T-95 .|) / 17}
$$

On the other hand, if the $R H$ is greater than $85 \%$ and the temperature is between 80 and 87 degrees $F$, then the following adjustment is added to $H I$ :

$$
\text { ADJUSTMENT }=[(R H-85) / 10] *[(87-T) / 5]
$$

The Rothfusz regression is not appropriate when conditions of temperature and humidity warrant a heat index value below about 80 degrees $\mathrm{F}$. In those cases, a simpler formula is applied to calculate values consistent with Steadman's results:

$$
H I=0.5 *\left\{T+61.0+[(T-68.0) * 1.2]+\left(R H^{*} 0.094\right)\right\}
$$

In practice, the formula is computed first and the result averaged with the temperature. If this above heat index value is 80 degrees $\mathrm{F}$ or higher, the full regression equation along with any adjustment as described above is applied.

\section{A transfer function model for the second order linear time invariant system}

When the temperature set point rises $0.5{ }^{\circ} \mathrm{C}$, the response of a population consisting of $\mathrm{n}$ ACs will be:

$$
D(t)=D_{s s}\left(\theta_{r e f}\right)+L^{-1}\left(G_{p}(s) 0.5 / s\right)
$$

Where $D_{s s}\left(\theta_{r e f}\right)$ is the asymptotic mean steady-state aggregated power demand of the AC group with constant temperature set point. It is also the duty cycle of the steady state. The term including $G_{p}(s)$, which belongs to a transient process of change, indicates the aggregated demand response of a $0.5{ }^{\circ} \mathrm{C}$ step in the form of a second-order linear model.

$$
D_{s s}(\theta)=\left(1+\frac{\log \left(1+\frac{H}{\theta_{a}-\theta-H / 2}\right)}{\log \left(1+\frac{H}{P R-\theta_{a}+\theta-H / 2}\right)}\right)^{-1}
$$

Where the hysteresis width $\mathrm{H}$ is obtained by the upper and lower limit of temperature which determines whether the $\mathrm{AC}$ is operating or switched off:

$$
H=\theta_{+}-\theta_{-}
$$

The second order transfer function model of an AC group is:

$$
G_{p}(s)=\frac{b_{2} s^{2}+b_{1} s+b_{0}}{s^{2}+2 \xi \omega_{n} s+\omega_{n}^{2}}
$$

Whose parameters are calculated from the equivalent thermal capacitance, thermal resistance, thermal power, hysteresis width and so on. The damping ratio $\xi$ and the undamped natural oscillation $\omega_{n}$ of the characteristic polynomials in the denominator are:

$$
\xi=\frac{\log (r)}{\sqrt{\pi^{2}+\log ^{2}(r)}}, \omega_{n}=\frac{\pi \mu_{v}}{\sqrt{1-\xi^{2}}},
$$

The coefficients in the numerator are:

$$
\begin{aligned}
b_{0} & =\frac{\omega_{n}^{2}\left(D_{s s}\left(\theta_{r e f}+0.5\right)-D_{s s}\left(\theta_{r e f}\right)\right)}{0.5}, \\
b_{1} & =0.5 \mu_{v}+2 b_{2} \xi \omega_{n}, b_{2}=D_{s s}\left(\theta_{r e f}\right),
\end{aligned}
$$

$$
r=\frac{\left|\operatorname{erf}\left(\frac{1}{0.9+2 \sqrt{2} \sigma_{r e l}}\right)-0.5\right|}{\left|\operatorname{erf}\left(\frac{1}{0.9}\right)-0.5\right|}
$$

where erf represents the Gaussian error function.

\section{A load control strategy for the AC group via the IMC controller}

Figure 1 shows a load control strategy for the AC group based on the internal model controller (IMC). The above second order transfer function model is used to design an IMC control structure to compute common offsets to a temperature set point to be broadcast to the ACs as a control signal.

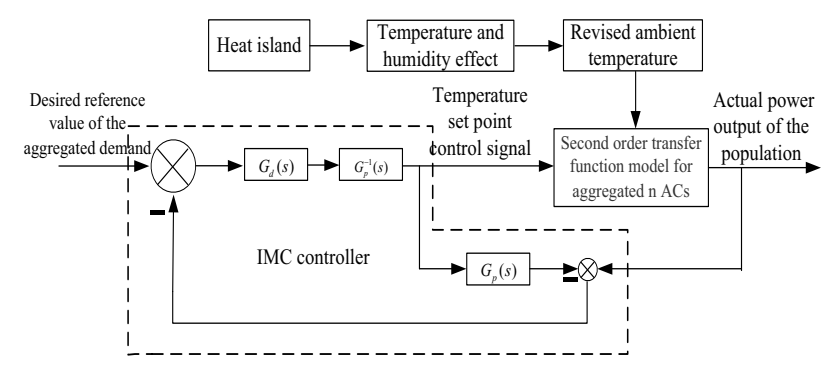

Figure 1. A load control strategy for the AC group via the IMC controller, according to the modified ambient temperature index based on the urban micro climate

The desired reference demand of the ACs minus the actual output power of them is the load tracking error, when the event of load control occurs. This load tracking error is taken as the input of the IMC controller. The heat island intensity is obtained by using the temperature inversion algorithm of the heat island effect. Then, the heat index is calculated by combining temperature and humidity. Based on the above microclimate, the ambient temperature index of urban central area is modified. From equations (12)- (19) it can be seen that, the parameters of the second order transfer function model for aggregated $n$ ACs vary with the ambient temperature. Consequently, the second order linear time invariant model is upgraded to the linear time varying model. The calculated temperature set point $u(t)$ is considered as the control signal of the air conditioning group.

The IMC controller contains a model of the control plant $G_{p}(s)$, its inverse $G_{p}^{-1}(s)$, and the target desired behaviour in the closed loop represented by its transfer function $G_{d}(s) . G_{p}(s)$ is the second order transfer function model of an AC group. For simplicity, we propose a first-order inertial element:

$$
G_{d}(s)=\frac{1}{T_{c} s+1}
$$

Where $T_{c}$ is the desired closed-loop time constant of the system. Because in air conditioning load control, reaching the steady state reference value within $10 \mathrm{~min}$ is reasonable, this paper chose $T_{c}=2 \mathrm{~min}$.

\section{Case study: demand shaping performance}


A population of ACs of similar characteristics can be regarded as a control group. We take a downtown area in Nanjing, China, as the research object where there are $18000 \mathrm{ACs}$ of 10000 residents participating in the demand response plan. Parameters of ACs in the group are as follows: thermal resistance $R \sim L N\left(2,0.4^{2}\right)$, thermal capacitance $\quad C \sim L N\left(3.6,0.72^{2}\right) \quad, \quad$ thermal power $P \sim L N\left(6,1.2^{2}\right)$. In this section, two scenario analytics by varying the temperature set point offset to control the aggregated power demand of $\mathrm{ACs}$ is carried out. Assuming that at the initial moment, $44.6 \%$ of the ACs are on, while the rest of them are off.

\subsection{Scenario 1: AC load control without considering urban microclimate}

In this scenario, the influence of urban microclimate on AC load control is neglected, and the parameters of the air conditioners remain unchanged, which are given in Table 1 .

Table 1. Parameters of the air conditioners.

\begin{tabular}{|c|c|c|}
\hline Parameters & Value & Description \\
\hline$R$ & $\mu_{R}=2{ }^{\circ} \mathrm{C} / \mathrm{kW}$ & $\begin{array}{c}\text { Equivalent thermal resistance, } \\
\text { log-normally distributed with mean } \\
\mu_{R} \text { and variance }\end{array}$ \\
\hline$P$ & $\mu_{C}=3.6$ \\
$\mathrm{kWh} /{ }^{\circ} \mathrm{C}$ & $\begin{array}{c}\text { Equivalent heat capacity log-normally } \\
\text { distributed with mean } \mu_{C} \text { and } \\
\text { variance } \sigma_{C}^{2}\end{array}$ \\
\hline$\theta_{a}$ & $32{ }^{\circ} \mathrm{C}$ & $\begin{array}{c}\text { Equivalent thermal power, log-normally } \\
\text { distributed with mean } \mu_{P} \text { and } \\
\text { variance } \sigma_{P}^{2}\end{array}$ \\
\hline$\theta_{+}$ & $20.5{ }^{\circ} \mathrm{C}$ & Higher end of hysteresis band \\
\hline$\theta_{-}$ & $19.5^{\circ} \mathrm{C}$ & Lower end of hysteresis band \\
\hline$\theta$ & $20{ }^{\circ} \mathrm{C}$ & Initial temperature set point for $n$ ACs \\
\hline$\sigma_{r e l}$ & 0.2 & defined as $\sigma_{r e l}^{2}=\sigma_{C}^{2} / \mu_{C}^{2}$ \\
\hline$\sigma_{C}^{2}$ & 0.4 & Variance of n ACs' heat capacity \\
\hline$\sigma_{R}^{2}$ & 0.72 & Variance of n ACs' thermal resistance \\
\hline$\sigma_{P}^{2}$ & 1.2 & Variance of n ACs' thermal power \\
\hline$H$ & $1{ }^{\circ} \mathrm{C}$ & Hysteresis width of indoor temperature \\
\hline $\begin{array}{c}C O P \\
\text { (coefficient } \\
\text { of } \\
\text { performance) }\end{array}$ & 2.5 & Load efficiency \\
\hline
\end{tabular}

From Equations (12) - (19) it can be seen that, when the number of air conditioning in the load control activity is determined, the total power of the aggregated air conditioning group is related to the ambient temperature. Combined with the equivalent thermal parameters (ETP) model of ACs, we make the following simulation: When the outdoor temperatures were respectively $[28,28.5,29$, $29.5,30,31,31.5,32,34,34.5,35,35.5,30.5,36]{ }^{\circ} \mathrm{C}$, assuming no control is applied, the baseline load power of the aggregated air conditioners is shown in Figure 2.

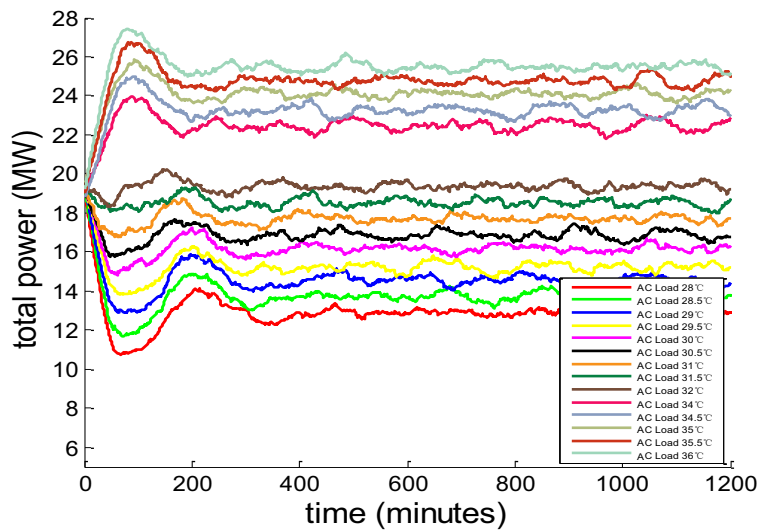

Figure 2. The power demand of the AC group at different outdoor temperatures

Figures 2 demonstrates that, without any control, the AC group will keep running independently. The profile of the aggregated demand of AC group is gentle, the duty cycle in steady state is 0.467 . Because the initial duty is set to 0.446 , the initial load of air-conditioning group is about $19 \mathrm{MW}$, the final load stabilized between 12 MW-26 MW. Such a conclusion can be drawn: The higher the outdoor temperature is, the higher the aggregate power of such population of ACs will be. On the contrary, the lower the outdoor temperature is, the lower the aggregate power of such population of ACs will be. When the ambient temperature is constant, assumed $32^{\circ} \mathrm{C}$, the simulated parameters are listed in Table 2, whose parameters are subsequently calculated by using Equations (12)-(19).

Table 2. Parameters of second order linear time invariant transfer function.

\begin{tabular}{|c|c|c|c|c|c|c|c|}
\hline Parameters & $\boldsymbol{D}_{\text {ss }}(\boldsymbol{\theta})$ & $\boldsymbol{r}$ & $\boldsymbol{\xi}$ & $\boldsymbol{\omega}_{\boldsymbol{n}}$ & $\begin{array}{c}\boldsymbol{b}_{\mathbf{0}} \times \\
\mathbf{1 0}^{-4}\end{array}$ & $\boldsymbol{b}_{\mathbf{1}}$ & $\boldsymbol{b}_{\mathbf{2}}$ \\
\hline Value & 0.429 & 0.431 & 0.259 & 0.033 & 0.378 & 0.012 & 0.429 \\
\hline
\end{tabular}

The whole load control scenario is from 12:00 p.m. to 16:00 p.m., during which the grid is in a peak load period. There is a power shortage and therefore needs a $20 \%$ reduction of air conditioning load. The load aggregator will raise the temperature set point of the ACs to achieve peak load shifting.

Figure 3 shows a comparison between the uncontrolled AC load, the desired reference value of the aggregated demand of a population of ACs for load shifting, and the actual output of ACs, when the outdoor temperature is constantly $32{ }^{\circ} \mathrm{C}$. The reason why the mismatch between the two figures exists only during the peak energy consumption lies in that, we only carry on the load control during peak hours and no control is performed outside this period. In Figure 3, the top blue curve is the 96 points AC load characteristic curve of the customers in the area on the maximum load day. We simulated the load values each second, by using the method of spline interpolation. Peak hours are from 12:00 a.m. to 16:00 p.m. During these periods, the electricity demand outstrips supply, and a power shortfall will appear. The top blue curve represents the aggregated power of 18,000 ACs without any control, the red dotted curve represents the desired $\mathrm{AC}$ load reference value for 
load shifting, and the solid black line represents the actual output power of AC load based on the proposed IMC controller and transfer function control model.

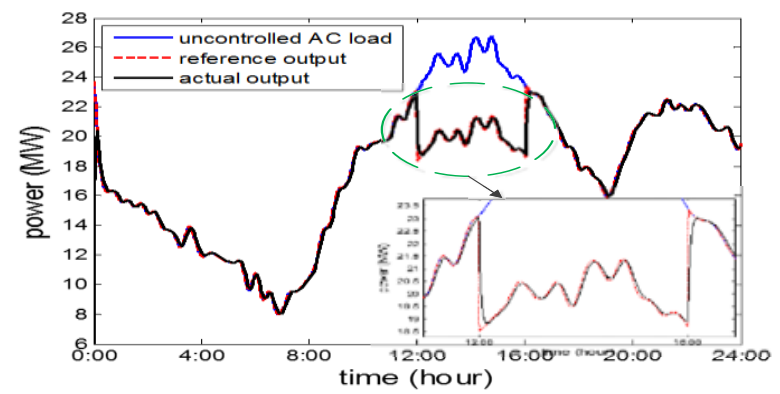

Figure 3. A comparison between the aggregated power of 18,000 ACs without any control, the desired AC load reference value for load shifting, and the actual output power of AC load based on the IMC controller, when the outdoor temperature is constantly $32{ }^{\circ} \mathrm{C}$

In this scenario, the second order transfer function of the AC group remains unchanged because of the constant ambient temperature. As can be seen from the figure, the effect of using the IMC controller is significantly good. Throughout the whole control period, the output power of the air conditionings has been following the reference value closely, and the tracking error is small. To some extent, the power shortage has been eased, and it meets with requires of rapidity, accuracy and stability of the control system.

\subsection{Scenario 2: AC load control considering the influence of urban microclimate}

In this scenario, the impact of urban microclimate on the air conditioning load control is considered. July 27 th, 2015 is selected as a typical day, the period of load control is still selected from 12:00 p.m. to 16:00 p.m., like the scenario 1. For during this period, Nanjing experiences the hottest weather and the maximum air conditioning load.

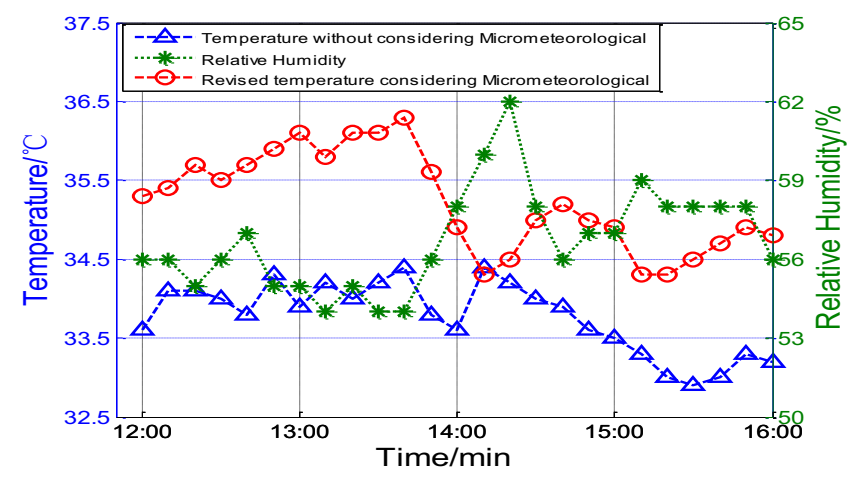

Figure 4. A comparison between the temperature before being modified (blue line) without considering urban microclimate, relative humidity (green line), and the modified temperature (red line) according to the influence of urban microclimate.

The heat island intensity is obtained by using the temperature inversion algorithm of the heat island effect. Then, the heat index is calculated by combining temperature and humidity. Based on the above microclimate, the ambient temperature index of urban central area is modified. Figure 4 shows a comparison between the temperature before being modified (blue line) without considering urban microclimate, relative humidity (green line), and the modified temperature (red line) according to the influence of urban microclimate. The real-time temperature and relative humidity on the same day, which was collected every $10 \mathrm{~min}$, was provided by the Jiangsu Provincial Meteorological Bureau.

In this scenario, we consider a more realistic situation, which means an ever-changing ambient temperature. It is dynamically modified in real time and constantly changing, depending on the weather conditions and urban microclimatic. Based on the expression of the second order transfer function model of the aggregate ACs (12)-(19), if the outdoor temperature varies with time, the second order transfer function model of the aggregated AC group will no longer be linear time invariant. Instead, it is changing with time. The model parameters vary with the outdoor temperature in the case of equivalent thermal resistance, thermal capacitance and other parameters remaining unchanged. Changes in the parameters of the second order transfer function of ACs are shown in Figure 5. The actual value of the ambient temperature was measured at weather stations on 15 August July 27th, 2015 , and the period is from 12:00 p.m. to 16:00 p.m..

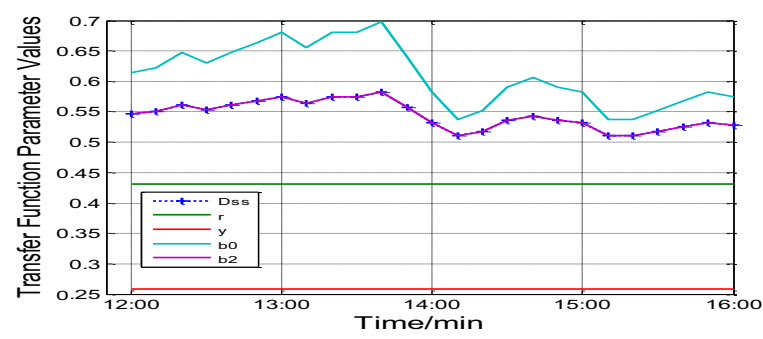

(a)

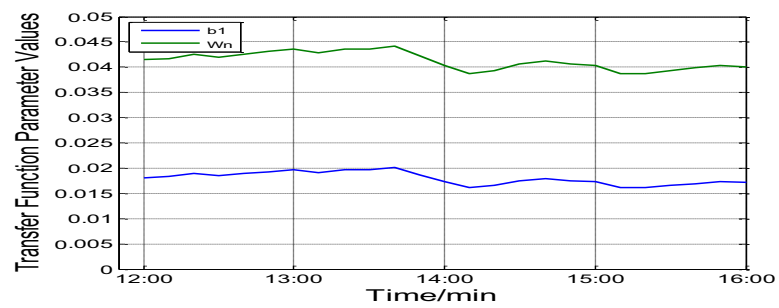

(b)

Figure 5. Changes in the parameters of the second order linear time varying transfer function model of ACs

In the simulation, the linear time invariant model of the aggregated ACs in Scenario 1 is changed into a time varying model. In Simulink simulation, if the parameters of the transfer function are changed, the response of the transfer function to the time domain is also changed. Subsequently, the effective length of the calculation may change, and this will result in a difficulty for simulink to calculate the transfer function with the ever-changing parameters.

The whole load control scenario is still from 12:00 p.m. to $16: 00$ p.m. Figure 6 illustrates the impact of urban microclimate on air-conditioning load control in the urban central area. The profiles are obtained by simulating the two order linear time invariant transfer function model, which is based on the parameters in Table 1 and the modified ambient temperature curve in Figure 4. The upper blue curve represents the aggregated baseline power of 18,000 ACs without any control, the middle red 
dashed curve is the desired $\mathrm{AC}$ load reference value for load shifting, the solid green curve is AC load curve obtained by the second order transfer function model for $\mathrm{n}$ ACs and the modified ambient temperature heat index based on urban microclimate, and the dashed black line at the bottom represents the AC load control effect without considering urban microclimate. A comparison of probability density distribution of the tracking error between the load control without considering urban microclimate and the load control considering urban microclimate is shown in Figure 7. As can be seen, without considering urban microclimate, the probability density is maximal when the load tracking error is 0 . In the case of considering urban micro climate in a more realistic scenario, the probability density is maximal when the load tracking error is $1.5 \mathrm{MW}$.

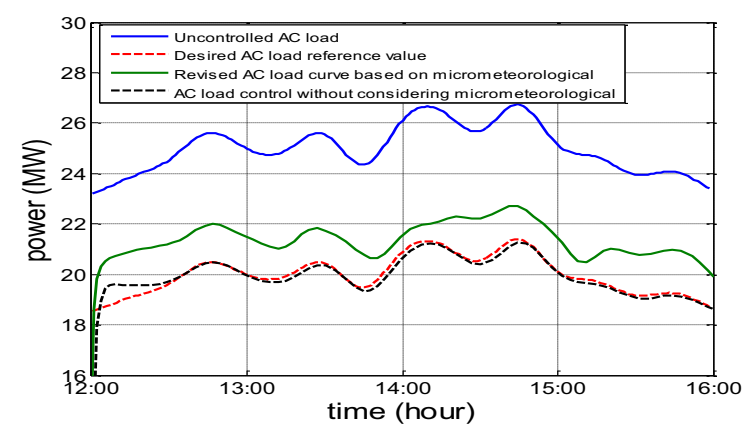

Figure 6. The impact of urban microclimate on air-conditioning load control in the urban central area.

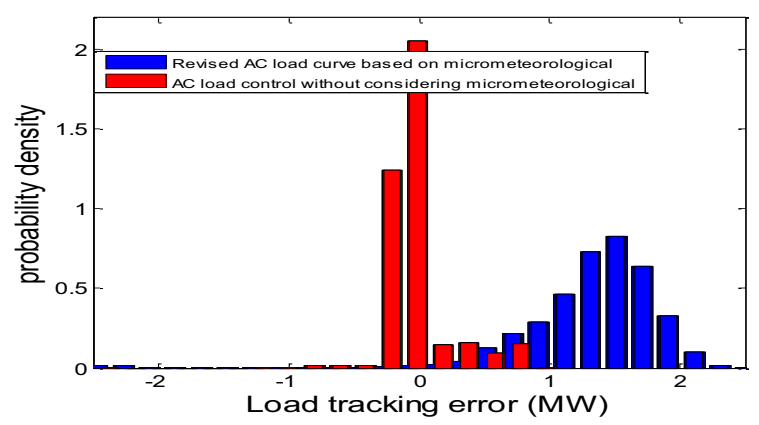

Figure 7. A comparison of probability density distribution of the tracking error between the load control without considering urban microclimate and the load control considering urban microclimate.

The performance of load control in the simulation is very good when the impact of the urban microclimate is not taken into account. Under the influence of urban microclimate, the maximum load of ACs participated in direct load control (DLC) is changed from 22.8MW to 21.2MW. But it is still far less than $26.8 \mathrm{MW}$ when there is no load control. As can be seen from the simulation results, because of the existence of urban microclimate, the potential of load control is reduced to $71.43 \%$ of the original.

\section{Conclusions}

The design of a closed-loop control strategy for AC loads to participate in load control was presented, and an IMC controller was also developed in this paper. Parameters of the model will be influenced by the ambient temperature and urban microclimate. The main contribution of this paper can be summarised as following: The heat island intensity is obtained by using the temperature inversion algorithm of the heat island effect. Then, the heat index is calculated by combining temperature and humidity. Based on the above urban microclimate, the ambient temperature index of urban central area is modified, and the second order linear time invariant model of aggregated ACs is upgraded to the linear time varying model. The consequent parameter changes of the second order transfer function model are studied and the influence of urban microclimate on $\mathrm{AC}$ load control is analyzed.

In fact, because of the inevitable existence of urban micro climate (e.g. heat island effect, the temperature and humidity effect), the apparent temperature is higher than the actual environmental temperature, resulting in an impact on the comfort of the occupants. Then the residents need to reduce the temperature set point of ACs to stay comfortable. As a result, the actual air-conditioning energy consumption rises, and the load control effect of ACs will be worse to some extent. From the simulation results it can also be seen that, the tendency of the AC load profile based on the proposed control model and control strategy is consistent with the desired AC load reference value.

\section{References}

1. C. Li, J. Zhou, Y. Cao, et al. Interaction between urban microclimate and electric air-conditioning energy consumption during high temperature season. Applied Energy, 117: 149-156 (2014).

2. C. Li, J. Shang, S. Zhu, et al. An analysis of energy consumption caused by air temperature affected accumulative effect of the air conditioning load. Automat Electric Power Syst, 34(20):30-32, (2010).

3. L. Yao, H. R. Lu. A second-way direct control of central air-conditioning load via the internet. IEEE Trans. on Power Delivery, vol. 24, no.1, 240-248 (2009).

4. T. Ojima, Changing Tokyo metropolitan area and its heat island model. Energy and Buildings, 15(1): 191-203,(1991).

5. M. Santamouris, N. Papanikolaou, I. Livada, et al. On the impact of urban climate on the energy consumption of buildings. Solar energy, 70(3): 201-216, (2001).

6. S. Hassid, M. Santamouris, N. Papanikolaou, et al. The effect of the Athens heat island on air conditioning load. Energy and Buildings, 32(2): 131-141(2000). 
7. X. T. Li. Effects of urbanization on cooling load of residential buildings. Heating Ventilating and Air Conditioning, 32(2): 79-81, (2002).

8. D. S. Callaway, I. A. Hiskens, Achieving Controllability of Electric Loads, Proceedings of the IEEE, 1, 184-199,(2011).

9. S. Kundu, N. Sinitsyn, S. Backhaus, and I. Hiskens, Modeling and Control of Thermostatically Controlled Loads. Available online: http://arxiv.org/abs/1101.2157 (accessed on 10 August 2015).

10. X. Hu,; B. Wang, S. Yang, T. Short, and L. A. Zhou, Closed-Loop Control Strategy for Air Conditioning Loads to Participate in Demand Response. Energies, 8, 8650-8681( 2015).

11. J. C. Jiménez-Muñoz, and J. A.Sobrino, A generalized single - channel method for retrieving land surface temperature from remote sensing data. Journal of Geophysical Research: Atmospheres (1984-2012), 108(D22),(2012).

12. L. P. Rothfusz, The heat index 'equation' (or, more than you ever wanted to know about heat index). NWS Technical Attachment SR90-23. NWS Southern Region Headquarters, Fort Worth, TX. 